\title{
Estimação de Parâmetros Genéticos de Características Reprodutivas em Suínos ${ }^{1}$
}

\author{
Aldrin Vieira Pires ${ }^{2}$, Paulo Sávio Lopes ${ }^{3,4}$, Robledo de Almeida Torres ${ }^{3,4}$, \\ Ricardo Frederico Euclydes ${ }^{3}$, André Ribeiro Corrêa da Costa ${ }^{2}$
}

\begin{abstract}
RESUMO - Com o objetivo de estimar os parâmetros genéticos e fenotípicos de características reprodutivas de suínos, foram utilizados dados de peso da leitegada ao nascimento (PLN), peso da leitegada ajustado para 21 dias (PL21), tamanho de leitegada ao nascimento (TLN), tamanho de leitegada ao desmame (TLD) e taxa de mortalidade (TM), de três raças de suínos, Duroc, Landrace e Large White. Variâncias e covariâncias genéticas e fenotípicas foram estimadas pelo Método da Máxima Verossimilhança Restrita (REML), usando modelo que inclui os efeitos genéticos direto e materno e comum de leitegada. Estes componentes foram usados para calcular as herdabilidades direta $\left(\mathrm{h}_{\mathrm{d}}^{2}\right)$, materna $\left(\mathrm{h}_{\mathrm{m}}^{2}\right)$ e total $\left(\mathrm{h}_{\mathrm{T}}^{2}\right)$, o efeito comum de leitegada $\left(\mathrm{c}^{2}\right)$ e as correlações genéticas e fenotípicas. As herdabilidades direta, materna e total apresentaram valores baixos a médios: 0,00 a $0,17,0,00$ a 0,14 e 0,00 a 0,15 , respectivamente, destacando a importância da seleção com base nas informações de parentes para o melhoramento genético destas características. As correlações entre os efeitos genéticos aditivos direto e materno foram, de modo geral, altas e negativas, evidenciando antagonismo entre estes efeitos. As estimativas de $\mathrm{c}^{2}$ foram em geral baixas para todas as características. As correlações genéticas entre PLN, PL21, TLN e TLD tenderam a ser altas e positivas, e as correlações entre estas características e TM apresentaram-se ora positivas, ora negativas.
\end{abstract}

Palavras-chave: efeito materno, leitegada, modelo animal, REML, suíno

\section{Genetic Parameters Estimation on Swine Reproductive Traits}

ABSTRACT - With the objective to estimate genetic and phenotypic parameters of swine reproductive traits, litter weight at birth (PLN), litter weight at 21 days (PL21), litter size at birth (TLN), litter size at weaning (TLD) and mortality rate (TM) data of three swine breeds, Duroc, Landrace and Large White were used. The genetic and phenotypic (co)variances were estimated by Restricted Maximum Likelihood (REML) method. These components were used to estimate the direct $\left(\mathrm{h}_{\mathrm{d}}^{2}\right)$, maternal $\left(\mathrm{h}_{\mathrm{m}}^{2}\right)$ and total $\left(\mathrm{h}_{\mathrm{T}}^{2}\right)$ heritabilities, the common litter effect $\left(\mathrm{c}^{2}\right)$, and the genetic and phenotypic correlations. The direct, maternal and total heritability estimates were small to medium, 0.00 to $0.17,0.00$ to 0.14 and 0.00 to 0.15 , respectively, indicating the necessity to include information on relatives in selection programs to improve these traits. The correlations among the additive direct and maternal genetic effects were, in general, high and negative, evidencing antagonism among these effects. The estimates of $\mathrm{c} 2$ were, in general, low, for all traits and breeds. The genetic correlations among PLN, PL21, TLN and TLD tended to be high and positive, and the correlations between these traits and TM were positive in some cases, and negative in others.

Key Words: animal model, litter, maternal effects, REML, swine

\section{Introdução}

O conhecimento das propriedades genéticas das populações baseia-se em parâmetros genéticos que podem ser obtidos utilizando-se componentes de variância, que podem ser estimados por vários métodos. O mais utilizado envolve o agrupamento dos indivíduos de acordo com o grau de parentesco entre si, a obtenção de componentes observacionais de variância e covariância (fenotípica) e a partição dos componentes observacionais em componentes causais, ou seja, variância e covariância relacionadas aos efeitos genéticos aditivo e materno, efeitos genéticos de dominância e de epistasia e efeitos ambientais permanentes e temporários (WILLHAM, 1963; FALCONER, 1981).

O conhecimento prévio dos componentes de variância e covariância é necessário na predição dos valores genéticos, principalmente quando se faz uso de métodos de predição como o BLUP (melhor predição linear não-viesada). Entretanto, estes componentes não são geralmente conhecidos e podem ser estimados por vários métodos, entre eles o da Máxima Verossimilhança Restrita (REML), recomendado para

\footnotetext{
${ }^{1}$ Parte da Tese do primeiro autor para obtenção do título de Mestre em Zootecnia.

2 MS em Zootecnia, UFV/DZO, Viçosa - MG, CEP: 36571-000. E.mail: avpires@alunos.ufv.br

3 Professor do DZO-UFV, Viçosa - MG, CEP: 36571-000.

4 Bolsista do CNPq.
} 
modelos lineares mistos e dados desbalanceados (MEYER, 1986).

A metodologia de modelos mistos para avaliação genética de suínos tem sido empregada e recomendada por vários pesquisadores em melhoramento animal (SORENSEN e KENNEDY, 1986; KEELE et al., 1988; LOPES, 1994a; MARTINS, 1995; e TORRES JR., 1996) e indicada por fornecer predições nãoviesadas de efeitos genéticos, efeitos comuns ou permanentes de ambiente e de grupo de animais, efeitos maternos e de endogamia, e efeitos de seleção, entre outros.

Entre os parâmetros genéticos, a herdabilidade $\left(\mathrm{h}^{2}\right)$ e a correlação genética são as principais estimativas de interesse para o planejamento de um programa de melhoramento.

As estimativas de herdabilidade para características reprodutivas são normalmente baixas, e a alta variação, observada na literatura, para estas estimativas é atribuída, principalmente, a fatores não-genéticos e genéticos não-aditivos. Modelos mais precisos devem ser desenvolvidos e propostos para obtenção de estimativas de parâmetros genéticos mais confiáveis, sobretudo para as características reprodutivas. Portanto, tão importante quanto estimar os parâmetros genéticos para uma população específica é, também, obter estimativas mais precisas dos componentes genéticos, pela inclusão de efeitos como o permanente de meio e o efeito genético aditivo materno, nos modelos de avaliação genética animal (PIRES, 1999).

Segundo LOPES et al. (1994b), muitos melhoristas têm dúvidas quanto à inclusão de características reprodutivas em melhoramento de suínos, em razão de suas baixas herdabilidades e de sua expressão ser limitada a animais adultos.

FREITAS et al. (1992) concluíram que a maior parte das características reprodutivas necessita de ajuste para ordem de parição e estação de parição, visando aumentar a precisão nos trabalhos de seleção.

Nos mamíferos, as mães exercem efeito maior que os pais sobre o fenótipo dos descendentes, pois, além da contribuição genética, podem influenciar suas progênies por meio do ambiente comum, pré e pós-natal, que lhes proporcionam. Assim, as características de crescimento, principalmente até o desmame, são determinadas pelo genótipo do próprio animal (efeito genético direto) e pelo genótipo de sua mãe (efeito genético materno).

Considerando-se que $\mathrm{P}_{\mathrm{x}}$ representa o valor fenotípico do indivíduo $\mathrm{X}$, influenciado por efeito materno, WILLHAM (1972) particionou Px em dois valores fenotípicos, um influenciado pelo valor genotípico de X e o outro pelo valor genotípico da mãe de $X$, representada por $W$. Representando os efeitos direto e materno por " $\mathrm{d}$ " e "m", respectivamente, então, $\mathrm{P}_{\mathrm{x}}=\mathrm{P}_{\mathrm{dx}}+\mathrm{P}_{\mathrm{mw}}$; em que $\mathrm{P}_{\mathrm{dx}}$ é o valor fenotípico para o efeito genético direto e Pmw, o valor fenotípico para o efeito materno. Fracionando o valor fenotípico nos componentes genético e ambientais, o fenótipo do indivíduo $\mathrm{X}$, filho da mãe $\mathrm{W}$, pode, então, ser representado por:

$$
\mathrm{P}_{\mathrm{x}}=\mathrm{G}_{\mathrm{dx}}+\mathrm{E}_{\mathrm{dx}}+\mathrm{G}_{\mathrm{mw}}+\mathrm{E}_{\mathrm{mw}}
$$

em que $\mathrm{G}_{\mathrm{dx}}$ é valor genotípico de $\mathrm{X}$ para o efeito direto; $\mathrm{E}_{\mathrm{dx}}$, valor ambiental de $\mathrm{X}$ para o efeito direto; $\mathrm{G}_{\mathrm{mw}}$, valor genotípico da mãe $\mathrm{W}$ para o efeito materno (herdável); $\mathrm{E}_{\mathrm{mw}}$, valor ambiental de $\mathrm{W}$ para o efeito materno, como ração, manejo, efeitos permanentes de meio etc (não-herdável).

Considerando ainda a existência de covariância entre os efeitos direto e materno $\left(\sigma_{\mathrm{GdGm}}\right)$, a variância fenotípica do indivíduo $\mathrm{X}\left(\sigma_{\mathrm{f}}^{2}\right)$ pode ser descrita como segue:

$$
\sigma_{\mathrm{f}}^{2}=\sigma_{\mathbf{G d}}^{2}+\sigma_{\mathbf{G m}}^{2}+\sigma_{\mathbf{G d G m}}+\sigma_{\mathbf{E d}}^{2}+\sigma_{\mathbf{E m}}^{2}
$$

No processo de decisão sobre quais características serão incluídas num programa de melhoramento, alguns fatores devem ser observados - importância econômica, facilidade de mensuração e correlações entre as características. As correlações genéticas e fenotípicas existentes entre as características devem ser consideradas, uma vez que a seleção exercida sobre determinada característica poderá influenciar outras características. Portanto, é importante que se conheçam estas correlações para que se possa planejar melhor as estratégias de seleção em programas de melhoramento de qualquer espécie.

Os objetivos do presente trabalho foram estimar os componentes de variância e covariância, para os efeitos genéticos direto e materno, e as correlações genéticas de características de leitegada em suínos.

\section{Material e Métodos}

Os dados utilizados neste trabalho são provenientes de animais da empresa COOPERCENTRAL, situada no município de Chapecó-SC, das raças Duroc, Landrace e Large White. Os animais da raça Duroc são provenientes de uma granja com capacidade de 120 matrizes. Os animais das raças Landrace e Large White são provenientes de duas granjas com capacidade de 160 e 240 matrizes cada. As informações 
1700 Rev. bras. zootec.

referem-se ao período de 1995 a 1998.

Ao nascimento, os leitões foram submetidos ao seguinte manejo: corte dos dentes, mossagem, pesagem e aplicação de ferro dextrano. Foi também realizada a padronização das leitegadas, sendo as transferências feitas somente dentro das raças, o que ocorreu apenas em cerca de $4 \%$ das leitegadas. A partir do décimo dia até uma semana após o desmame, os leitões receberam ração pré-inicial. A maioria dos animais foi pesada aos 21 dias de idade. Os animais que por algum motivo não foram pesados aos 21 dias tiveram seus pesos ajustados para esta idade de acordo com a seguinte fórmula:

$\mathrm{PAD}=(((\mathrm{POD}-1,4) / \mathrm{ID}) * 21)+1,4$

em que PAD é peso ajustado do leitão para 21 dias; $\mathrm{POD}$, peso observado do leitão na pesagem à aproximadamente 21 dias; ID, idade do leitão no dia da pesagem; e 1,4, peso padrão adotado para o peso ao nascer.

O desmame foi feito entre 24 e 28 dias de idade, não sendo feita a pesagem ao desmame.

Após o desmame, os animais foram levados para creche, classificados por peso e colocados em baias para 20 animais. As baias possuíam $20 \%$ do piso ripado, onde se localizavam os bebedouros, e $80 \%$ de piso de concreto sólido com cobertura escamoteável e lâmpada para o aquecimento dos animais nas primeiras semanas de creche, principalmente no inverno. Da segunda semana até o final da creche, os animais receberam ração inicial à base de milho e soja.

As características estudadas foram: tamanho da leitegada ao nascer (TLN), tamanho de leitegada ao desmame (TLD), peso da leitegada ao nascer (PLN), peso da leitegada corrigido para os 21 dias de idade (PL21) e taxa de mortalidade do nascimento ao desmame (TM).

As estimativas dos componentes de (co)variância foram obtidas pelo REML, utilizando o programa MTDFREML (BOLDMAN et al., 1995). Para a estimação das herdabilidades direta e materna, bem como da correlação entre os efeitos genéticos direto e materno, foram utilizados os componentes de variância e covariância obtidos a partir de análise de característica única e, para as estimativas de correlações genéticas, foram feitas análises conjuntas das características, avaliando-as duas a duas. O seguinte modelo foi usado para as análises:

$$
\mathbf{y}=\mathbf{X b}+\mathbf{Z}_{1} \mathbf{d}+\mathbf{Z}_{2} \mathbf{m}+\mathbf{Z}_{3} \mathbf{p}+\mathbf{e}
$$

em que y é vetor de observações; $\mathbf{X}=$ matriz de incidência de efeitos fixos; $b$, vetor de efeitos fixos, $\mathbf{Z}_{\mathbf{1}}=$ matriz de incidência de efeitos genéticos aditivos diretos; $\underset{\sim}{\mathrm{d}}$, vetor de efeitos genéticos aditivos diretos; $\mathbf{Z}_{\mathbf{2}}$, matriz de incidência de efeitos genéticos aditivos maternos; $\underset{\sim}{m}$, vetor de efeitos genéticos aditivos maternos; $\mathbf{Z}_{\mathbf{3}}$, matriz de incidência de efeitos comuns de leitegada; $\mathrm{p}$, vetor de efeitos comuns de leitegada; $\underset{\sim}{\mathrm{e}}$, vetor de efeito residual.

O critério de convergência utilizado foi $-2 \log _{\mathrm{e}} \mathrm{da}$ verossimilhança inferior a $10^{-9}$. Após cada convergência, o programa foi reiniciado, usando as estimativas obtidas anteriormente como valores iniciais.

Admitiu-se que y tem distribuição normal multivariada,

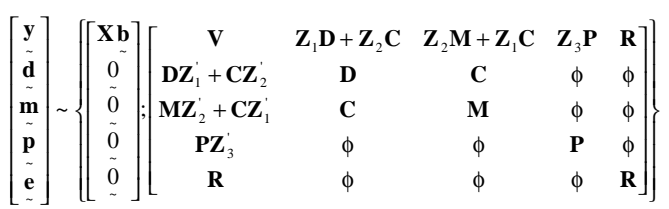

em que $\mathbf{V}=\mathbf{Z}_{1} \mathbf{D Z} \mathbf{Z}_{1}+\mathbf{Z}_{2} \mathbf{M Z _ { 2 }}+\mathbf{Z}_{1} \mathbf{C Z _ { 2 }}+\mathbf{Z}_{2} \mathbf{C Z}_{1}^{\prime}+\mathbf{Z}_{3} \mathbf{P Z}+\mathbf{R} ; \mathrm{D}=\mathrm{A}$ $\otimes \mathrm{D}_{0} ; \mathrm{M}=\mathrm{A} \otimes \mathrm{M}_{0} ; \mathrm{C}=\mathrm{A} \otimes \mathrm{C}_{0} ; \mathrm{P}=\mathrm{I} \otimes \mathrm{P}_{0} ;$ $\mathrm{R}=\mathrm{I} \otimes \mathrm{R}_{0} ; \mathbf{D}_{\mathbf{0}}=$ matriz de (co)variâncias genéticas aditivas diretas; $\mathbf{M}_{\mathbf{0}}=$ matriz de (co)variâncias genéticas aditivas maternas; $\mathbf{C}_{\mathbf{0}}=$ matriz de covariâncias genéticas aditivas entre os efeitos direto e materno; $\mathbf{P}_{\mathbf{0}}=$ matriz de (co)variâncias atribuídas aos efeitos comuns de leitegada; $\mathbf{R}_{\mathbf{0}}=$ matriz de (co)variâncias residuais; $\mathbf{A}=$ matriz de numeradores dos coeficientes de parentesco de Wright entre os animais; $\phi=$ matriz de zeros; $I$ = matriz identidade; $\mathrm{e}$ $\otimes=$ operador do produto direto.

Quando se trata de análises de característica única, as matrizes $\mathbf{D}_{\mathbf{0}}, \mathbf{M}_{\mathbf{0}}, \mathbf{C}_{\mathbf{0}}, \mathbf{P}_{\mathbf{0}}$ e $\mathbf{R}_{\mathbf{0}}$ foram substituídas por $\hat{\sigma}_{d}^{2}, \hat{\sigma}_{m}^{2}, \hat{\sigma}_{d m}, \hat{\sigma}_{p}^{2} \mathrm{e} \hat{\sigma}_{\mathrm{e}}^{2}$, em que $\hat{\sigma}_{d}^{2}$ é estimador da variância atribuída aos efeitos genéticos diretos; $\hat{\sigma}_{m}^{2}$, estimador da variância atribuída aos efeitos genéticos maternos; $\hat{\sigma}_{\mathrm{dm}}$, estimador da covariância entre os efeitos genéticos direto e materno; $\hat{\sigma}_{\mathrm{p}}^{2}$, estimador da variância atribuída aos efeitos comuns de leitegada; e $\hat{\sigma}_{e}^{2}$, estimador da variância atribuída aos efeitos residuais.

A herdabilidade total dos efeitos genéticos aditivos $\left(\hat{\mathrm{h}}_{\mathrm{T}}^{2}\right)$ foi calculada de acordo com WILLHAM (1972):

$$
\hat{\mathrm{h}}_{\mathrm{T}}^{2}=\left(\hat{\sigma}_{\mathrm{d}}^{2}+0,5 \hat{\sigma}_{\mathrm{m}}^{2}+1,5 \hat{\sigma}_{\mathrm{dm}}\right) / \hat{\sigma}_{\mathrm{f}}^{2}
$$

em que $\hat{\sigma}_{f}^{2}=$ estimador da variância fenotípica; demais termos definidos anteriormente.

Efeito fixo de grupo contemporâneo foi considerado nos modelos, sendo que para as raças Landrace e Large White era constituído por rebanho, ano e estação de parição; para a raça Duroc considerava apenas os efeitos de ano e estação de parição. O efeito da covariável ordem de parto, linear (registros 
PIRES et al.

1701

das três primeiras parições) para Duroc e quadrático (registros das quatro primeiras parições) para Landrace e Large White, foi incluído nos modelos. Estações de parição foram classificadas em: 1 -verão (dezembro a fevereiro), 2 - outono (março a maio), 3 - inverno (junho a agosto) e 4 - primavera (setembro a novembro).

\section{Resultados e Discussão}

As médias e os desvios-padrão das características para cada raça são apresentadas na Tabela 1 . Destacam-se as médias de peso de leitegada ao nascimento (PLN), das raças Duroc e Landrace e as médias de peso da leitegada aos 21 dias (PL21), para as raças Landrace e Large White. Quanto a tamanho de leitegada ao nascimento (TLN) e ao desmame (TLD), as três raças apresentaram médias muitos próximas. Entretanto, a raça Large White foi a que apresentou maior taxa de mortalidade $(9,30 \%)$.

As estimativas das variâncias genética aditiva direta $\left(\hat{\sigma}_{d}^{2}\right)$ e materna $\left(\hat{\sigma}_{m}^{2}\right)$, a covariância entre os efeitos genéticos direto e materno $\left(\hat{\sigma}_{\mathrm{dm}}\right)$, a variância de efeitos comuns de leitegada $\left(\hat{\sigma}_{\mathrm{p}}^{2}\right)$, a variância residual $\left(\hat{\sigma}_{\mathrm{e}}^{2}\right)$ e a variância fenotípica $\left(\hat{\sigma}_{\mathrm{f}}^{2}\right)$, para cada raça, são apresentadas na Tabela 2 .

As estimativas de herdabilidades para os efeitos genéticos aditivos direto $\left(\hat{\mathrm{h}}_{\mathrm{d}}^{2}\right)$ e materno $\left(\hat{\mathrm{h}}_{\mathrm{m}}^{2}\right)$, de correlações entre os efeitos aditivos direto e materno $\left(r_{d m}\right)$, de herdabilidade total $\left(\hat{\mathrm{h}}_{\mathrm{T}}^{2}\right)$, da proporção atribuída aos efeitos comuns de leitegada $\left(\hat{\mathrm{c}}^{2}\right)$ e respectivos erros-padrão (EP) são apresentados na Tabela 3.

Observando-se os resultados, verifica-se que as estimativas de herdabilidade direta de todas as características, para as três raças, foram próximas às encontradas na literatura. As estimativas de herdabilidade para peso da leitegada ao nascimento (PLN) e peso da leitegada aos 21 dias (PL21) foram 0,05 e 0,$08 ; 0,04$ e 0,13 ; e 0,17 e 0,04 , para as raças Duroc, Landrace e Large White, respectivamente. Estes valores foram semelhantes àqueles encontrados por ALVES (1986), os quais variaram de -0,18 a 0,24 para PLN e de 0,11 a 0,20 para PL21, embora o autor tenha utilizado a correlação entre meio-irmãos paternos em seu trabalho. PARK e KIM (1986) encontraram valores de herdabilidades para PLN, nas raças Landrace, Yorkshire, Duroc e Hampshire, de 0,32; 0,32; 0,23; e 0,09, respectivamente.

As estimativas de herdabilidade para tamanho de leitegada ao nascimento (TLN) e tamanho de leitegada ao desmame (TLD) foram 0,03 e 0,$14 ; 0,15$ e 0,11 ; e
Tabela 1 - Número de registros, porcas, médias e desviospadrão das características

Table 1 - Number of registers, dams, averages and standard errors of traits

\begin{tabular}{|c|c|c|c|c|c|}
\hline \multirow[b]{2}{*}{ Item } & \multicolumn{5}{|c|}{ Características (Trait) } \\
\hline & $\begin{array}{l}\text { PLN } \\
(\mathrm{kg})\end{array}$ & $\begin{array}{c}\text { PL21 } \\
(\mathrm{kg})\end{array}$ & $\begin{array}{l}\text { TLN } \\
\text { (Unid.) }\end{array}$ & $\begin{array}{l}\text { TLD } \\
\text { (Unid.) }\end{array}$ & $\begin{array}{l}\mathrm{TM} \\
(\%)\end{array}$ \\
\hline & \multicolumn{5}{|c|}{ Duroc } \\
\hline $\begin{array}{l}\text { Média } \\
\text { Average }\end{array}$ & 15,38 & 45,23 & 9,32 & 8,63 & 8,14 \\
\hline $\begin{array}{l}\text { Desvio-padrão } \\
\text { Standard error }\end{array}$ & 3,64 & 11,15 & 2,21 & 1,59 & 10,85 \\
\hline $\begin{array}{l}\text { № de registros } \\
\text { Number of records }\end{array}$ & 604 & 554 & 604 & 554 & 559 \\
\hline № de porcas & 335 & & & & \\
\hline $\begin{array}{l}\text { Number of dams } \\
\text { № animais em A } \\
\text { Number of animals in } A\end{array}$ & 6843 & & & & \\
\hline & \multicolumn{5}{|c|}{ Landrace } \\
\hline $\begin{array}{l}\text { Média } \\
\text { Average }\end{array}$ & 15,22 & 54,27 & 9,54 & 8,91 & 8,37 \\
\hline $\begin{array}{l}\text { Desvio-padrão } \\
\text { Standard error }\end{array}$ & 4,01 & 12,44 & 2,79 & 1,74 & 11,58 \\
\hline $\begin{array}{l}\text { № de registros } \\
\text { Number of records }\end{array}$ & 699 & 688 & 727 & 656 & 715 \\
\hline $\begin{array}{l}\text { № de porcas } \\
\text { Number of dams }\end{array}$ & 367 & & & & \\
\hline $\begin{array}{l}\text { № animais em } \mathrm{A} \\
\text { Number of animals in } \mathrm{A}\end{array}$ & 8655 & & & & \\
\hline
\end{tabular}

Number of animals in $A$

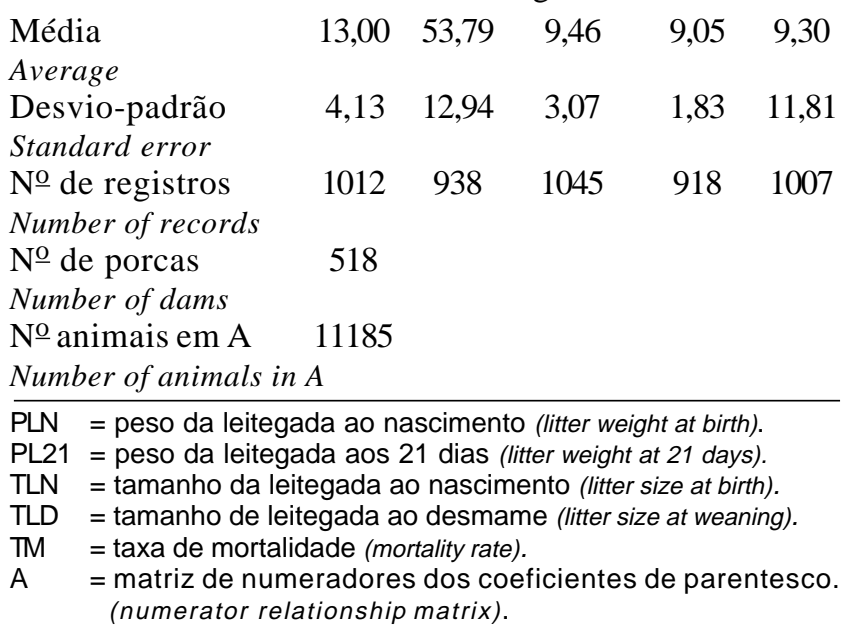

0,16 e 0,03, para as raças Duroc, Landrace e Large White, respectivamente. UPNMOOR (1984) obteve herdabilidade para tamanho de leitegada ao nascimento de 0,17 e aos 21 dias, de 0,18. PARK e KIM (1986) relataram, para as raças Landrace, Yorkshire, Duroc e Hampshire, valores para TLN de 0,20; 0,25; 0,04 ; e 0,12 , respectivamente. BABOT et al. (1994) obtiveram estimativas para tamanho de leitegada, em uma população de Large White, variando entre 0,081 e 0,092 e para uma população de Landrace, entre 
1702 Rev. bras. zootec.

Tabela 2 - Estimativas das variâncias genéticas aditivas diretas $\left(\hat{\sigma}_{\mathrm{d}}^{2}\right)$, materna $\left(\hat{\sigma}_{\mathrm{m}}^{2}\right)$, covariância entre os efeitos genéticos direto e materno $\left(\hat{\sigma}_{\mathrm{dm}}\right)$, variância atribuída aos efeitos comuns de leitegada $\left(\hat{\sigma}_{\mathrm{p}}^{2}\right)$, variância residual $\left(\hat{\sigma}_{\mathrm{e}}^{2}\right)$ e variância fenotípica $\left(\hat{\sigma}_{\mathrm{f}}^{2}\right)$, obtidas da análise de característica única

Table 2 - Estimates of direct $\left(\hat{\sigma}_{\mathrm{d}}^{2}\right)$ and maternal $\left(\hat{\sigma}_{\mathrm{m}}^{2}\right)$ additive genetic variance, covariance between the direct and maternal effects $\left(\hat{\sigma}_{\mathrm{dm}}\right)$, common litter effects $\left(\hat{\sigma}_{\mathrm{p}}^{2}\right)$, residual variance $\left(\hat{\sigma}_{\mathrm{e}}^{2}\right)$ and phenotypic variance $\left(\hat{\sigma}_{\mathrm{f}}^{2}\right)$, in single trait analyses

\begin{tabular}{|c|c|c|c|c|c|c|}
\hline $\begin{array}{l}\text { Características } \\
\text { Trait } \\
\end{array}$ & $\hat{\sigma}_{\mathrm{d}}^{2}$ & $\hat{\sigma}_{\mathrm{m}}^{2}$ & $\hat{\sigma}_{\mathrm{dm}}$ & $\hat{\sigma}_{\mathrm{p}}^{2}$ & $\hat{\sigma}_{\mathrm{e}}^{2}$ & $\hat{\sigma}_{f}^{2}$ \\
\hline \multicolumn{7}{|c|}{ Duroc } \\
\hline PLN & 0,59519 & 0,39600 & 0,48549 & 1,34356 & 9,69674 & 12,51698 \\
\hline PL21 & 9,55288 & 0,02733 & 0,51098 & 13,91160 & 92,11899 & 116,12183 \\
\hline TLN & 0,12886 & 0,29563 & 0,19518 & 0,47431 & 3,68051 & 4,77449 \\
\hline TLD & 0,34239 & 0,00132 & $-0,02126$ & 0,12865 & 2,04074 & 2,49184 \\
\hline $\mathrm{TM}$ & 11,69347 & 0,25961 & $-1,74233$ & 5,91135 & 93,11099 & 109,23308 \\
\hline \multicolumn{7}{|c|}{ Landrace } \\
\hline PLN & 0,50664 & 1,95665 & $-0,99564$ & 1,44417 & 10,82805 & 13,73986 \\
\hline PL21 & 16,88987 & 0,36290 & $-2,47575$ & 5,59231 & 111,17112 & 131,54046 \\
\hline TLN & 1,06668 & 0,61752 & $-0,21219$ & 0,00004 & 5,50908 & 6,98114 \\
\hline TLD & 0,31641 & 0,12451 & $-0,15447$ & 0,00002 & 2,57944 & 2,86591 \\
\hline $\mathrm{TM}$ & 0,63118 & 1,71798 & 1,04132 & 1,55958 & 122,72585 & 127,67591 \\
\hline \multicolumn{7}{|c|}{ Large White } \\
\hline PLN & 2,52698 & 0,24986 & $-0,79460$ & 2,17062 & 10,99833 & 15,15119 \\
\hline PL21 & 5,31495 & 2,83828 & 3,88393 & 11,73030 & 125,45059 & 149,21806 \\
\hline TLN & 1,43959 & 0,09952 & $-0,37850$ & 0,61470 & 7,30538 & 9,08068 \\
\hline TLD & 0,08761 & 0,10274 & $-0,06719$ & 0,27841 & 2,86884 & 3,27041 \\
\hline $\mathrm{TM}$ & 0,12703 & 0,00804 & $-0,03197$ & 11,77970 & 123,77544 & 135,65821 \\
\hline
\end{tabular}

PLN = peso da leitegada ao nascimento (litter weight at birth).

PL21 = peso da leitegada aos 21 dias (litter weight at 21 days)

TLN = tamanho da leitegada ao nascimento (litter size at birth).

TLD = tamanho de leitegada ao desmame (litter size at weaning).

$\mathrm{TM}=$ taxa de mortalidade (mortality rate).

0,053 e 0,056 . SHORT et al. (1994) encontraram 0,14 para herdabilidade de TLN.

ROBISON (1972) relatou que as estimativas de herdabilidade para tamanho de leitegada são baixas, e afirmou que a correlação genética negativa entre os efeitos genéticos materno (ou efeito ambiental comum) e direto poderia explicar parte destes resultados. No presente trabalho, as covariâncias entre os efeitos genéticos direto e materno $\left(\hat{\sigma}_{\mathrm{dm}}\right)$ foram predominantemente negativas, exceto para a raça Duroc, as quais foram negativas apenas para as características tamanho de leitegada ao desmame e taxa de mortalidade. Covariância negativa, entre os efeitos genéticos direto e materno, também foi encontrada por IRGANG et al. (1994a e 1994b).

A inclusão de outros efeitos aleatórios, além do genético aditivo direto e residual, causa redução de $\hat{\sigma}_{e}^{2}$, porque as frações da variância, atribuídas aos efeitos genético aditivo materno e permanente de meio, quando não são consideradas, são então acu- muladas junto à variância residual, fazendo com que esta apresente maiores valores.

PIRES (1999) observou que, à medida que se incluíram outros efeitos aleatórios (materno e, ou, comuns de leitegada) no modelo de avaliação genética, havia, de modo geral, redução das estimativas de $\sigma_{d}^{2}$, indicando que, ao não se considerarem tais efeitos aleatórios no modelo, obtêm-se estimativas viesadas de componentes de variância, o que poderia levar à classificação errônea dos animais pelo BLUP.

Em geral, as estimativas de herdabilidade da mortalidade de leitões foram baixas, 0,$11 ; 0,00$; e 0,00 para Duroc, Landrace e Large White, respectivamente. Estes valores estão dentro do intervalo, 0,0 a 0,22, obtido por UPNMOOR (1984).

As estimativas de herdabilidade encontradas foram, de modo geral, baixas, fazendo necessária a utilização da informação de parentes para se alcançar maior êxito no melhoramento genético destas características. 
PIRES et al.

Tabela 3 - Estimativas de herdabilidades para os efeitos genéticos aditivos direto $\left(\hat{\mathrm{h}}_{\mathrm{a}}^{2}\right)$ e materno $\left(\hat{\mathrm{h}}_{\mathrm{m}}^{2}\right)$, de correlações entre os efeitos aditivos direto e materno $\left(r_{d m}\right)$, de herdabilidade total $\left(\hat{\mathrm{h}}_{\mathrm{T}}^{2}\right)$, da proporção atribuída aos efeitos comuns de leitegada $\left(\hat{\mathrm{c}}^{2}\right)$ e respectivos erros-padrão (EP), obtidas da análise de característica única

Table 3 - Estimates of direct $\left(\hat{\mathrm{h}}_{\mathrm{d}}^{2}\right)$ and maternal $\left(\hat{\mathrm{h}}_{\mathrm{m}}^{2}\right)$ heritabilities, correlation between the direct and maternal effects $\left(r_{d m}\right)$, total heritability $\left(\hat{\mathrm{h}}_{\mathrm{T}}^{2}\right)$, proportion attributed to permanent environment $\left(\hat{\mathrm{c}}^{2}\right)$, and respective standard errors, in single trait analyses

\begin{tabular}{|c|c|c|c|c|c|}
\hline $\begin{array}{l}\text { Característica } \\
\text { Trait }\end{array}$ & $\hat{\mathrm{h}}_{\mathrm{d}}^{2}+\mathrm{EP}$ & $\hat{\mathrm{h}}_{\mathrm{m}}^{2}+\mathrm{EP}$ & $r_{d m}+\mathrm{EP}$ & $\hat{\mathrm{h}}_{\mathrm{T}}^{2}$ & $\hat{\mathrm{c}}^{2}+\mathrm{EP}$ \\
\hline & \multicolumn{5}{|c|}{ Duroc } \\
\hline PLN & $0,05+0,069$ & $0,03+0,039$ & $1,00+0,067$ & 0,12 & $0,110+0,067$ \\
\hline PL21 & $0,08+0,073$ & $0,00+0,010$ & $1,00+* * * *$ & 0,09 & $0,790+0,065$ \\
\hline TLN & $0,03+0,068$ & $0,06+0,064$ & $1,00+1,463$ & 0,12 & $0,099+0,078$ \\
\hline TLD & $0,14+0,115$ & $0,00+0,070$ & $-1,00+* * * *$ & 0,12 & $0,052+0,104$ \\
\hline \multirow[t]{2}{*}{$\mathrm{TM}$} & $0,11+0,104$ & $0,00+0,220$ & $-1,00+5,723$ & 0,08 & $0,054+0,092$ \\
\hline & \multicolumn{5}{|c|}{ Landrace } \\
\hline PLN & $0,04+0,111$ & $0,14+0,079$ & $-1,00+2,326$ & 0,00 & $0,110+0,084$ \\
\hline PL21 & $0,13+0,107$ & $0,00+0,024$ & $-1,00+5,499$ & 0,10 & $0,040+0,082$ \\
\hline TLN & $0,15+0,111$ & $0,09+0,070$ & $-0,26+0,746$ & 0,15 & $0,000+0,080$ \\
\hline TLD & $0,11+0,101$ & $0,04+0,069$ & $-0,78+1,671$ & 0,05 & $0,000+0,083$ \\
\hline \multirow[t]{2}{*}{ TM } & $0,00+0,067$ & $0,01+0,051$ & $1,00+5,420$ & 0,02 & $0,010+0,059$ \\
\hline & \multicolumn{5}{|c|}{ Large White } \\
\hline PLN & $0,17+0,128$ & $0,02+0,019$ & $-1,00+1,817$ & 0,10 & $0,140+0,053$ \\
\hline PL21 & $0,04+0,063$ & $0,02+0,043$ & $1,00+1,077$ & 0,08 & $0,079+0,063$ \\
\hline TLN & $0,16+0,099$ & $0,01+0,013$ & $-1,00+1,447$ & 0,10 & $0,068+0,064$ \\
\hline TLD & $0,03+0,053$ & $0,03+0,046$ & $-0,71+2,215$ & 0,01 & $0,085+0,062$ \\
\hline $\mathrm{TM}$ & $0,00+0,056$ & $0,00+0,038$ & $-1,00+* * * *$ & 0,00 & $0,087+0,061$ \\
\hline
\end{tabular}

PLN = peso da leitegada ao nascimento (litter weight at birth).

PL21 = peso da leitegada aos 21 dias (litter weight at 21 days).

TLN = tamanho da leitegada ao nascimento (litter size at birth).

TLD = tamanho de leitegada ao desmame (litter size at weaning).

$\mathrm{TM}=$ taxa de mortalidade (mortality rate).

As estimativas de $c^{2}$ foram baixas para todas as características e raças, variando entre 0,000 e 0,140, à exceção de PL21 na raça Duroc, que foi de 0,790 ; semelhantes às encontradas por IRGANG et al. (1994a), que variaram de 0,000 a 0,096.

As estimativas de correlações genéticas para as características em estudo encontram-se na Tabela 4.

Verifica-se que as estimativas de correlações genéticas entre as características peso da leitegada ao nascer (PLN), peso da leitegada aos 21 dias (PL21), tamanho de leitegada ao nascer (TLN) e tamanho de leitegada ao desmame (TLD), para as raças Duroc e Landrace, foram positivas e relativamente altas, variando de 0,51 (PLN e TLD) a 1,00 (PL21 a TLN) para Duroc e de 0,41 (PL21 e TLN) a 0,93 (TLN e TLD) para Landrace. A raça Large White apresentou resultados semelhantes, exceto a estimativa entre PL21 e TLD, que foi negativa, na ordem de $-0,04$, as demais variaram de 0,10 (TLN e PL21) a 0,61 (PLN e TLN). As estimativas de correlações envolvendo a taxa de mortalidade (TM) e as demais características tenderam ser negativas na raça Duroc, exceto entre TM e PLN, que foi 0,29; negativas também para a raça Large White, exceto entre TLN e TM, que foi igual a 0,45. A raça Landrace apresentou estimativas de correlações genéticas positivas entre todas as características.

Estes resultados indicam que devem ser utilizadas metodologias ou procedimentos que não desprezem tais correlações, mas a análise destas cinco características simultaneamente certamente levaria a problemas computacionais. Nesse caso, uma alternativa seria descartar as informações relativas às características PLN e PL21 e considerar apenas TLN, TLD e TM, uma vez PLN e PL21 são altamente e positivamente correlacionadas com TLN e TL21. A combinação de TLN, TLD e TM, em um único índice, pode ser uma boa opção para se buscar, com maior eficiência, otimizar o ganho genético do rebanho.

A natureza das características em estudo pode ter influenciado os resultados. Estas características de leitegada são referidas como se fossem da porca, mãe da leitegada; no entanto, na constituição genética destas, não está somente o efeito genético da 
1704 Rev. bras. zootec.

Tabela 4 - Estimativas de correlações genéticas para as características, analisadas duas a duas

Table 4 - Estimates of genetic correlation for the traits, in two traits analyses

\begin{tabular}{|c|c|c|c|c|}
\hline \multirow[b]{2}{*}{$\begin{array}{l}\text { Características } \\
\text { Traits }\end{array}$} & \multicolumn{4}{|c|}{$\begin{array}{c}\text { Correlações genéticas } \\
\text { Genetic correlations }\end{array}$} \\
\hline & PL21 & TLN & TLD & $\mathrm{TM}$ \\
\hline & \multicolumn{4}{|c|}{ Duroc } \\
\hline PLN & 0,74 & 0,66 & 0,51 & 0,29 \\
\hline PL21 & & 1,00 & 0,65 & $-0,68$ \\
\hline TLN & & & 0,80 & $-0,66$ \\
\hline \multirow[t]{2}{*}{ TLD } & & & & $-0,92$ \\
\hline & \multicolumn{4}{|c|}{ Landrace } \\
\hline PLN & 0,61 & 0,89 & 0,68 & 0,57 \\
\hline PL21 & & 0,41 & 0,67 & 0,29 \\
\hline TLN & & & 0,93 & 0,93 \\
\hline \multirow[t]{2}{*}{ TLD } & & & & 0,36 \\
\hline & \multicolumn{4}{|c|}{ Large White } \\
\hline PLN & 0,49 & 0,61 & 0,12 & $-0,04$ \\
\hline PL21 & & 0,10 & $-0,04$ & $-0,87$ \\
\hline TLN & & & 0,45 & 0,45 \\
\hline TLD & & & & $-0,61$ \\
\hline
\end{tabular}

PLN = peso da leitegada ao nascimento (litter weight at birth).

PL21 = peso da leitegada aos 21 dias (litter weight at 21 days).

TLN = tamanho da leitegada ao nascimento (litter size at birth).

TLD = tamanho de leitegada ao desmame (litter size at weaning).

$\mathrm{TM}$ = taxa de mortalidade (mortality rate) .

porca, e sim o somatório dos efeitos dos leitões que compõem a leitegada, o qual, por sua vez, é constituído metade pelo efeito direto da mãe e metade do pai. Quando se avalia a leitegada, como característica da mãe, está se desprezando a contribuição do cachaço, pai da leitegada, que exerce influência tanto no tamanho quanto no peso da leitegada. ROSO e SEVERO (1997) verificaram que o cachaço, pai da leitegada, é importante fonte de variação para número de leitões nascidos vivos.

Uma possível maneira de se contornar esse problema da natureza da característica seria avaliar o peso individual do leitão ao nascimento e ao desmame. Dessa forma, o animal estaria sendo avaliado por seu próprio mérito genético. A característica agora seria o peso do leitão, em que o efeito genético aditivo direto envolvido é o do próprio indivíduo, e não o da leitegada, em que há um somatório de efeitos genéticos dos filhos, que são atribuídos à mãe. Entretanto, do ponto de vista prático, essa análise teria pouca utilidade, visto que ao produtor interessa o tamanho e peso da leitegada, e não do leitão. Além disso, a característica deixaria de ser da fêmea (reprodutiva) e passaria a ser exclusivamente do leitão.

\section{Conclusões}

As estimativas de herdabilidade foram baixas a médias, indicando ser necessária a utilização da informação de parentes para se alcançar maior êxito no melhoramento genético de características reprodutivas, o que pode ser feito utilizando a metodologia BLUP.

A existência de correlações genéticas entre as características reprodutivas indica que devem utilizadas, na avaliação destas, metodologias ou procedimentos que não desprezem tais correlações.

A combinação de TLN, TLD e TM, em um único índice, pode ser boa opção para se otimizar o ganho genético, por considerar as correlações entre as características reprodutivas.

\section{Agradecimento}

À COOPERCENTRAL, pela cessão dos dados, o que possibilitou a execução deste trabalho.

\section{Referências Bibliográficas}

ALVES, R.G.O. Estudo genético de características reprodutivas em suínos e avaliação de curvas de crescimento em cruzamentos dialélicos. Viçosa, MG: UFV, 1986. 124p. Tese (Doutorado em Zootecnia) - Universidade Federal de Viçosa, 1986

BABOT, D., NOGURA, J.L., ALFONSO, L., et al. Influence of management effects and comparison group size on the prodiction of breeding values for litter size in pigs. In: WORLD CONGRESS ON GENETICS APPLIED TO LIVESTOCK PRODUCTION, 5, 1994, Guelph, Ontario, Canada. Proceedings... Guelph: University of Guelph, 1994. v.17, p.351-354.

BOLDMAN, K.G., KRIESE, S., VAN VLECK, L.D., et al MTDFREML Users guide USDA/ARS Claycenter, Nebrasca, 1995. $114 \mathrm{p}$.

FALCONER, D.S. 1981. Introdução à genética quantitativa. Tradução de SILVA, M.A., SILVA, J.C. Viçosa, MG: UFV, Impr. Univ. 279p.

FREITAS, R.T.F., OLIVEIRA, A.I.G., LIMA, J.A.F. et al 1992. Estudo de características reprodutivas em matrizes de criações de suínos no Sul do Estado de Minas Gerais. R. Soc. Bras. Zootec., 21(2):186-199.

IRGANG, R., FÁVERO, J.A., KENNEGY, B.W. 1994a. genetic parameters for litter size of different parities in Duroc, Landrace, and Large White sows. J. Anim. Sci., 72(9): 22372246.

IRGANG, R., FÁVERO, J.A., KENNEDY, B.W. Variâncias genética e de ambiente comum e prolificidade em diferentes parições de porcas Duroc, Landrace e Large White. In: REUNIÃO ANUAL DA SOCIEDADE BRASILEIRA DE ZOOTECNIA, 31, 1994, Maringá. Anais... Maringá: SBZ, 1994 b, p.249.

KEELE, J.W., JONHSON, R.K., YOUNG, L.D. 1988. Comparison of methods of predicting breeding values of swine. J. Anim. Sci., 66(12):3040-3048. 
PIRES et al.

LOPES, P.S. Avaliação genética de suínos utilizando metodologia de modelos mistos. Viçosa: UFV, 98 p. 1994. Tese (Doutorado em Zootecnia) - Universidade Federal de Viçosa, 1994a.

LOPES, P.S., FREITAS, R.T.F., FERREIRA, A.S. 1994b. Melhoramento de suínos. Viçosa, MG: UFV, 39p.

MARTINS, E.N. Desenvolvimento de uma estratégia computacional para a seleção de coelhos usando a melhor predição linear não-viesada. Viçosa, MG: UFV, 1995. 117p. Dissertação (Doutorado em Zootecnia) - Universidade Federal de Viçosa, 1995.

MEYER, K. 1986. Between algorithms: A "Short Cut" restricted maximum likelihood procedure to estimate variance components. J. Dairy Sci., 69(7):1904-1916.

PARK, Y.I., KIM, J.B. Heritabilities of litter size and litter weight at birth in swine. In: WORLD CONGRESS ON GENETICS APPLIED TO LIVESTOCK PRODUCTION, 3, 1986, Lincoln, Nebraska, USA. Proceedings... Lincoln: University of Nebraska, 1986. v.10, p.59-62.

PIRES, A.V. Avaliação genética de características reprodutivas em suínos. Viçosa, MG: Universidade Federal de Viçosa, 1999. 83p. Dissertação (Mestrado em Zootecnia) - Universidade Federal de Viçosa, 1999.

ROBISON, O.W. 1972. The role of maternal effects in animal breeding: V. Maternal effects in swine. J. Anim. Sci., 35(6):1303-1315.

ROSO, V.M., SEVERO, J.L.P. Efeito da ordem do parto sobre o número de leitões nascidos vivos em leitegadas Landrace $\mathrm{x}$ Large White. In: REUNIÃO ANUAL DA SOCIEDADE BRASILEIRA DE ZOOTECNIA, 34, 1997, Juiz de Fora. Anais... Juiz de Fora: SBZ, 1997, v.3, p.314-316.

SHORT, T.H., WILSON, E.R., MCLAREN, D.G. Relationships between growth and litter traits in pig dam lines. In: WORLD CONGRESS ON GENETICS APPLIED TO LIVESTOCK PRODUCTION, 5, Guelph, Ontario, Canada, 1994. Proceedings... Guelph: University of Guelph, 1994, v.17, p.413-416.
SORENSEN, D.A., KENNEDY, B.W. 1986. Analysis of selection experiments using mixed model methodology. J. Anim. Sci., 63(1):245-258.

TORRES Jr., R.A.A. Eficiência das informações de diferentes grupos contemporâneos na avaliação genética de suínos utilizando modelos mistos em procedimentos uni $e$ multivariados. Viçosa, MG: UFV, 1996. 117p. Dissertação (Mestrado em Genética e Melhoramento) - Universidade Federal de Viçosa, 1996.

UPNMOOR, I. Influência de fatores genéticos e de ambiente sobre a produtividade de suínos mestiços Landrace e Large White. Viçosa, MG: UFV, 1984. 180p. Dissertação (Mestrado em Zootecnia) - Universidade Federal de Viçosa, 1984.

WILLHAM, R.L. 1963. The covariance between relatives for characters composed of components contributed by related individuals. Biometrics, 19(1):18-26.

WILLHAM, R.L. 1972. The role of maternal effects in animal breeding: III. Biometrical aspects of maternal effects in animals. J. Anim. Sci., 35(6):1288-1293.

Recebido em: 17/06/99

Aceito em: 02/06/00 